manner in which a vaginal examination was spoken of by one of the gentlemen present at the Society. I think the challenge of Dr. Bennet should have been accepted at once, and that a committee should have been, and should now be, appointed, to test the existence or the non-existence of the thousand and one "ulcers" or "abrasions," of which so much has been said of late.

The gentleman to whom I have alluded above, huffed the idea of indecency in making a vaginal examination. There need be no exposure of the person of the patient; surgeons make no scruple about an examination of the rectum, (as if the two examinations could, morally speaking, be compared.) But, if there be no exposure of the person, and if the examination of the rectum be frequently made, is there, at first, no wounding of the feelings, and is there, afterwards, no deterioration and blunting of those feelings, by the repeated daily or weekly use of the speculum vaginæ in the virgin, and in the very young even amongst the married? I loudly proclaim that there is such deterioration, and that the female who has been subjected to such treatment is not the same person in delicacy and purity that she was before.

I have known cases of the most revolting attachment, on the part of such patients, to the practice and to the practitioner. I have known them to speak of "the womb" and of "the uterine organs" with a familiarity which was formerly unknown, and which, I trust, will ere long be obsolete. The current of the ideas becomes hypochondricalily directed to these organs. The very mind is poisoned. A new and lamentable form of hysteria, I had almost said, of furor uterinus, is induced, with this aggravation, that the subject of distress is either concealed by the greatest effort, or explained at the expense of virgin or female modesty.

There is a case of "poisoned mind" in the male sex, induced by the quack doings of the day, relative to the existence of impotency, which all of us must have treated and deplored. A similar case of "mental poisoning" is now being induced in the other sex by the frequent, constant, and undue reference, on the part of the profession, (?) to the condition of "the uterine organs."

These latter patients become reserved, and moody, and perverse, and speak unintelligibly in broken sentences; the peace and happiness of the family circle is broken up; subjects are discussed on the domestic hearth which ought never to be mentioned except in the sick-room; words which wound are spoken, and thoughts which are derogatory are expressed, by other, perhaps by the male, members of the family.

One poor miserable patient comes to me weekly, thus afficted. She had been treated by the speculum and the caustic for months, as an out-patient at University College Hospital. I sent her to Dr. Robert Lee twice. Twice that gentleman examined and declared that there was no uterine or vaginal disease. Meanwhile, the miserable patient's mind is absorbed by this ideal malady, and the peace of her husband's home is destroyed.

I sent another patient to Dr. Robert Lee a few days ago, (whom I had never seen,) under similar circumstances, but moving in a different rank of life. The same opinion was given, the miserable patient suffering dire disappointment!

I recently attended a poor curate's wife, who had come to Iondon for medical aid, at, as I suppose, great inconvenience. During my short attendance, this patient was constantly urged by a friend, a titled lady, (the aristocracy always take the lead in quackery, to send for her physician, who is a strong abettor of the speculum. The course which followed may be imagined, and need not be described. A case of more complicated misery for a husband cannot well be conceiveda sickly wife, afflicted with uterine hypochondriasis, set upon by a titled adrocate of the uterine quackery, with straitened resources.

The advocates of the speculum speak of cases which had resisted the efficacy of the usual general and local treatment, and which yielded to the use of the speculum and the caustic. I have seen cases in which, the speculum and caustic having been employed-and unduly employed, as I believe-the patient remained more miserably afflicted in mind and body than ever, and this the effect of that treatment. Whether the former supposition be as well founded as the latter, I will not presume to determine; but I believe the cases in which the young, and especially the unmarried, are afflicted so as really to justify the use of the speculum, to be rare; and the cases in which the injection of a solution of the nitrate of silver by her own hand may not take the place of the application of this valuable remedy in substance by the hand of the practitioner, to be rare indeed.
I will not advert even to the epithets which have been applied to the frequent use of the speculum by our French neighbours, who are so skilled in these matters; but $I$ will ask, what father amongst us, after the details which $I$ have given, would allow his virgin daughter to be subjected to this "pollution"? Let us, then, maintain the spotless dignity of our profession, with its well-deserved character for purity of morals, and throw aside this injurious practice with indignant scorn, remembering that it is not mere exposure of the person, but the dulling of the edge of the virgin modesty, and the degradation of the pure minds, of the daughters of England, which are to be avoided.

\section{NOTE ON A MODE OF EXCITLNG DEGLUTITION IN CERTAIN CASES.}

By W. FREDERICK BARLOW, Esq., M.R.C.S.

I AM desirous of directing attention to an ingenious mode of producing deglutition, first devised and employed by $\mathbf{M r}$. Simpson, of Stamford.*

In a case of puerperal convulsion, this gentleman having put some fluid into the mouth of his patient, found that no swallowing happened; but on dashing some cold water on the face, the desired event followed instantly.

What is the rationale of this phenomenon, which $\mathbf{I}$ have now on some occasions brought about myself? and has it any value in practice?

I have always noticed, on exciting deglutition in this way, that it was preceded by an inspiration, and I suppose the explanation of its occurrence to be simply this:- The act of inspiration has the effect of drawing the fluid backwards to the pharynx, or causing it to be there conveyed. When this happens, it must be swallowed in the generality of cases; even if the patient be ever so refractory, he cannot prevent his own deglutition if the fluid but reach where his volition has no power.

I have remarked the good result of this mode of producing swallowing under two circumstances, chiefly.

1st. Where a patient, though there be fluid in his mouth, refuses to swallow, he may be obliged to do so by a sudden dash of cold water on his face. $\mathrm{He}$ is unprepared for the stimulus, and it will act. But the practitioner must use caution to effect his end, for everything may depend on the application being sudden, and the patient being quite off his guard.

$2 n d$. I have seen the plan of no little value in the convulsions of children. It is sometimes most desirable to make them swallow before the convulsions have subsided, supposing them dependent on irritation of the stomach, as they often are. To wait until they are completely over, may be, sometimes, to risk life. I have known blood drawn in these circumstances, and so, to irritation, was needlessly added perilous exhaustion. In several cases, I have introduced between the almost perpetnally clenched teeth an emetic solution; but because of the continuance of the expiratory efforts, it was not swallowed. In this difficulty I have found the full, abrupt application of cold to the face of most essential service; a free inspiration, and, afterwards, an act of deglutition ensued. So one reflex action brought about another.

It is, of course, known to every practitioner, that when drink is put into the mouth, the obvious reason of its not being swallowed is its not being carried far enough backwards. There are instances, it is true, in which we cannot so place it. Convulsive efforts will sometimes effectually prevent us doing so, unless we use a degree of violence, which, in the case of young children, would be particularly well avoided. Let Mr. Simpson's contrivance have a trial in this difficulty. Perchance a full inspiration will accomplish more than awkward force. Besides, the inspiration will, of itself, do good, even though swallowing should not follow; for deep and perfect inspirations are the happiest of all omens, looking to the immediate consequences of a fit. So long as efforts at expiration continue, there is a plain aberration of the motor force, and a danger of serious congestion of the brain and cord. Ever let it be remembered by every one who would treat convulsions properly, that inspiration tends to empty, expiration to fill, the veins.

But as to the production of vomiting in convulsions, it is hard sometimes to overrate its importance. An irritant should be removed in some cases as rapidly as, because it may

* See The Lancer for December, 1842, p. 437, and Dr. Marshall Hall's "New Memoir on the Nervous System," p. 84 
be no less destructive than, a deadly poison. Some of the most violent and exaggerated forms of convulsion which $I$ have seen, though they proved quite curable, persisted obstinately until the stomach was relieved. To prescribe an emetic in some cases of this kind, and then leave its administration to some ignorant attendant, is often, virtually, to write pro form $\hat{a}$; she will, perhaps, succeed in insinuating a little of it between the teeth-a useless thing, so long as the respiration remain expiratory.

Any method of exciting deglutition, not generally used, is to be regarded as deserving of attention; nor is it to be despised for simplicity, but the rather to be valued on that account. Dr. Tyler Smith, in a recently published case of uterine hæmorrhage, adopted the plan of causing deglutition, which I have shortly treated of. I doubt not that there are many circumstances under which it would be found available and expedient ; but I will not here refer to more than those in which experience has assured me that it may be of use.

Westminster Hospital, April, 1850.

\section{REPORT OF A CASE OF CHOREA SUCCESS- FULLY TREATED BY BLISTERING AND THE TOPICAL APPLICATION OF BELLADONNA.}

Bx SAMUEL MAULT, Esq., Surg,; Fenny Stratford, Bucks.

ON looking over your "Mirror of Medicine and Surgery of the London Hospitals" in The LanceT of May 18, I was much interested in the account of the two cases of Chorea treated by Dr. Barker in St. Thomas's Hospital.

The following case occurred to me whilst assisting a medical gentleman in Glasgow. And since the treatment adopted proved very successful, should you think it worthy of record you will oblige me by inserting it in a corner of your valuable journal.

I have endeavoured (from copious notes taken at the time) to compress the most important features of the case in the smallest number of words.

CASE.-Agnes Campbell,aged fourteen, a dark-complexioned, thin, unhealthy looking child, was seized with chorea on the 19 th of February, 1849. States that for some time previously she has suffered from a very relaxed and disordered state of the bowels; but within the last week her bowels have become obstinately costive. About four days ago she complained of excessive pain in the wrist, elbow, and shoulder joints, but there was no heat or swelling about them. She took a smart purgative, which, as soon as it began to act, relieved her of the pain.

On the morning of the $19 \mathrm{th}$, she began to experience involuntary twitching of the muscles of the arm and forearm; this gradually increased, and in fortyeight hours the paroxysms became very severe; her arms were thrown about in every possible direction, and her legs were constantly either drawn up, or jerked out at full length with all her strength; sometimes these irregular movements extended themselves to the muscles of the back and sides, and made her writhe and con tort herself, so that she presented a most singular appearance.

As soon as these violent paroxysms came on, it was found necessary to secure her in bed, or she would have thrown herself out every few minutes.

At times she assumed the most frightful and hideous aspect, for not only was there involuntary and irregular movements, and twitchings in the muscles of the arm, trunk, and lower extremities, but the muscles of the face participated in the general disturbance, and caused her to contort her face into every imaginable shape. As soon as these movements relaxed, her face assumed a most foolish expression.

On the 19th, the morning on which the twitching of the muscles first commenced, she took three compound colocynth pills, and these acted pretty freely on the bowels, and she discharged several copious and offensive motions. The same day, she commenced taking a mixture of quinine, and sulphate of iron, which she continued taking for three days without any abatement of the symptoms. This mixture was then exchanged for one composed of decoction of cinchona, with Fowler's solution, and this was persevered in for other four days, and still there was no improvement whatever; in fact, the paroxysms increased in intensity and duration. I was now resolved to adopt the following plan, having seen it used with complete success in arresting convulsions having. their origin from another cause.

A blister, about an inch in breadth, and eight inches in length, was applied along the course of the spine-in twelve hours after, when it was well risen, the cuticle was removed, and a thin plaster of belladonna was applied, (the plaster-was made by spreading a thin layer of the watery extract of belladouna on lint.) In half an hour after its application there was a manifest improvement, and within four hours the convulsions ceased, and there was scarcely any movement of the muscles, and the child lay quietly dosing.

She remained free from convulsions about thirty-nine hours when slight twitchings recommenced about the mouth and lower jaw. Another dressing of belladonna was now applied, and had the desired effect of removing the twitchings. On the fifth day after the application of the blister, another blister was applied, accompanied with a dressing of the belladonna; owing to slight convulsive movements recurring in the left arm, this re-application of the dressing stayed the movements, and from this time the child rapidly recovered.

It is necessary for me to state that the belladonna-dressing was kept on for a period of an hour each dressing.

I must likewise state that my patient continued taking Fowler's solution during the whole time the above applications were used, and owing to a torpid condition of the bowels, smart purgatives were administered occasionally.

May, 1850 .

\section{foreign 琋epartment.}

\section{ACADEMY OF MEDICINE OF PARIS.}

Influence of Albumen in the Blood.

Messrs. Becquerel and Rodier have addressed to the Academy a paper, entitled, "On Anæmia from a Diminished Proportion of Albumen in the Blood, and on the Dropsies consequent on that change." We subjoin a summary, and the conclusions of this communication:-1. Just as there is an anæmia caused by the diminished proportion of the globules of the blood, so ought a pathological state to be admitted which is characterized by a diminution of albumen in the blood.2 . 'This lessening of the albumen in the serum of the blood may take place rapidly; its symptoms are then, paleness; a yellowish tint of the face; great debility, and above all, general anasarca, without albumen in the urine.-3. A great number of acute dropsies, still looked upon as essential, ought manifestly to be attributed to this pathogenic cause.-4. The diminution of albumen in the serum may take place slowly. It then constitutes a chronic pathological state, which is characterized by the symptoms just stated, (2.)-5. Most dropsies, formerly called essential or passive, must be referred to this alteration.-6. The diminution of the albumen of the blood, produced either rapidly or slowly, is quite independent of the diminution of the globules. These two alterations of the blood exist, however, frequently together; sometimes the one and sometimes the other predominating. -7 . The lower proportion of the globules cannot bring on dropsy, except a diminution of albumen of the serum has likewise taken place.8. The symptoms which are added to those above quoted, when the diminution of globules is combined with the loss of albumen, are-a bellows-murmur with the first sound of the heart, a continuous bruit in the jugular veins, an intermittent one in the carotids, dyspnoa, and palpitations.-9. The causes which may bring on a rapid or a slow loss of albumen in the blood, are--insufficient food, considerable hæmorrhage, prolonged diarrhœea, and marshy exhalations.-10. The same effects are produced by organic affections, such as those of the heart, and Bright's disease; they then constitute a cachectic state of the patient.-11. The pathological state to which the name cachexia is generally given is nothing else but the aggregate of the symptoms which result from the diminished proportion of albumen, connected with a lesser amount of globules in the blood. The first of these causes accounts for the dropsies which are frequent in cachexia, for the discoloration of the skin, and the great debility of the patients. The second explains the bellows murmur, be it either cardiac or vascular; the dyspnoea, palpitations, \&c.-12. The preceding distinctions exercise a great influence on the diagnosis, prognosis, and treatment of these dropsies. (The committee appointed to report on this paper is composed of Messrs. Andral, Grisolle, and Jolly.) 